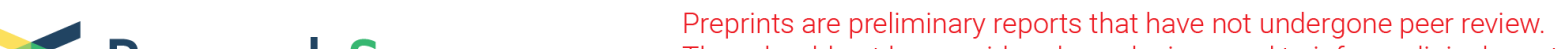

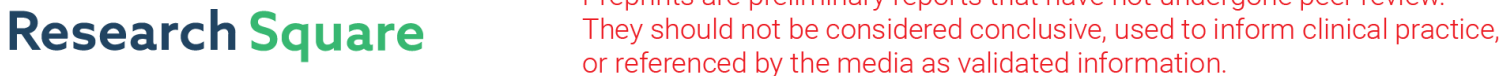

\section{Plasmonics-Nanofluidics Metamaterial: An \\ Ultrasensitive Platform for Terahertz biosensing and Quantitative Measurement of Molecules}

\section{Mingzhen Xie}

Lanzhou University First Affiliated Hospital

Jinchun He

Lanzhou University First Affiliated Hospital

Yu Liu

Third Military Medical University Southwest Hospital

Huiyan Tian

Third Military Medical University Southwest Hospital

\section{Xinyu Zhan}

Third Military Medical University Southwest Hospital

\section{Ke Xia}

Third Military Medical University Southwest Hospital

\section{Mengya Li}

Third Military Medical University Southwest Hospital

\section{Lamy de la Chapelle Marc}

UMR6283: Institut des Molecules et Materiaux du Mans

\section{Weiling Fu}

Third Military Medical University Southwest Hospital

Yang Zhang ( $\sim$ millen001@163.com )

third military medical university https://orcid.org/0000-0002-4061-4408

\section{Research}

Keywords: THz spectroscopy, microfluidic chip, metamaterial sensor, liquid-phase sensing

Posted Date: December 7th, 2020

DOI: https://doi.org/10.21203/rs.3.rs-120675/v1

License: (c) (1) This work is licensed under a Creative Commons Attribution 4.0 International License.

Read Full License 


\section{Abstract}

Background: The terahertz spectroscopy has attracted enormous attention for label free chemical and biological sensing, due to the presence of molecular fingerprints and remote sensing capabilities. However, because of the strong absorption of THz waves in aqueous solutions, detecting liquid-phase biological samples by $\mathrm{THz}$ spectroscopy has encountered strong constraints.

Results: Here, we demonstrate for the first time a rapid and label-free sensing for liquid-phase biological samples using THz spectroscopy based on microfluidic metasensors. The metasensors were integrated with microfluidic chip to precisely control the liquid sample thickness within 220 nanometer (MMS-N) and 50 micrometer (MMS-U). The metasensors include a periodic split ring resonator (SRR) with two resonant peaks in the $\mathrm{THz}$ range. The sensitivity(S) and corresponding figure of merit (FOM) of the two resonance peaks of MMS-N and MMS-U are analyzed with respect to different refractive index matching solution. Both the two resonance peaks of the metasensors are sensitive to the varies refractive index environment and high-frequency resonant of MMS-N shows the best sensitivity $(136 \mathrm{GHz} / \mathrm{RIU})$. In addition, the labelfree biological sensing capability was conducted through qualitative and quantitative LDL and ox-LDL, where a shift of resonance frequency was observed as the LDL and ox-LDL concentration increased.

Conclusions: The results indicated nanofluidic metasensor presented excellent sensing capability for liquid-phase molecular analysis by THz spectroscopy, and the proposed sensing system has the potentials to be developed as a rapid, label-free and highly sensitive detection tool for molecular analysis by THz sensing.

\section{Full Text}

This preprint is available for download as a PDF.

\section{Figures}


(a)

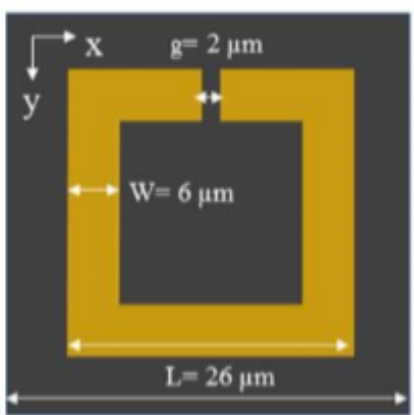

$\mathrm{T}=40 \mu \mathrm{m}$

(d)
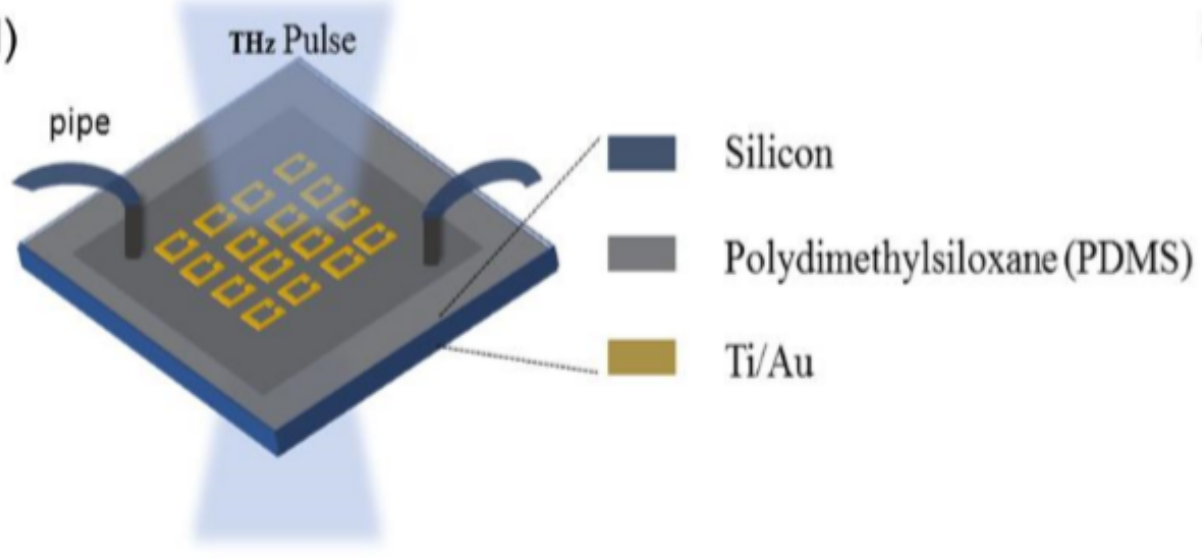

(b)

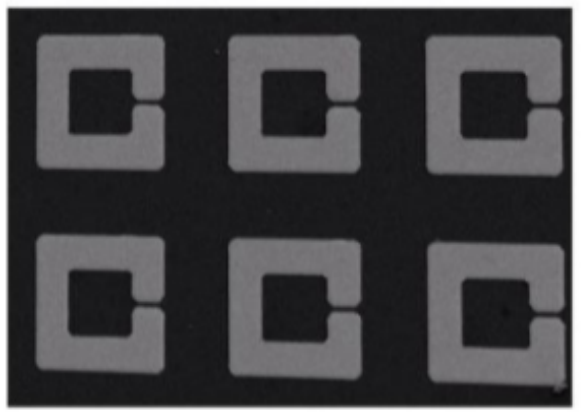

(c)

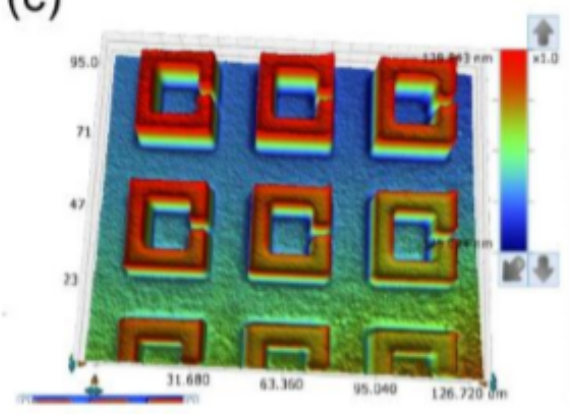

(e)

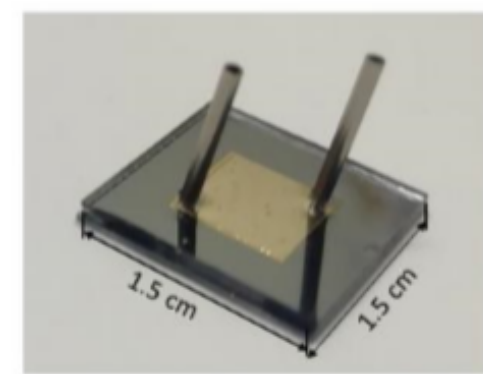

Figure 1

The schematic and characterization of fabricated MMS. (a) The Schematic of SRR. (b) Scanning electron microscope characterization of SRRs. (c) 3D topography of white light interferometer of SRRs. (d) Schematics of the ideal THz MMS and its cross-sectional device structure. (e) MMS made by microelectro-mechanical processing.
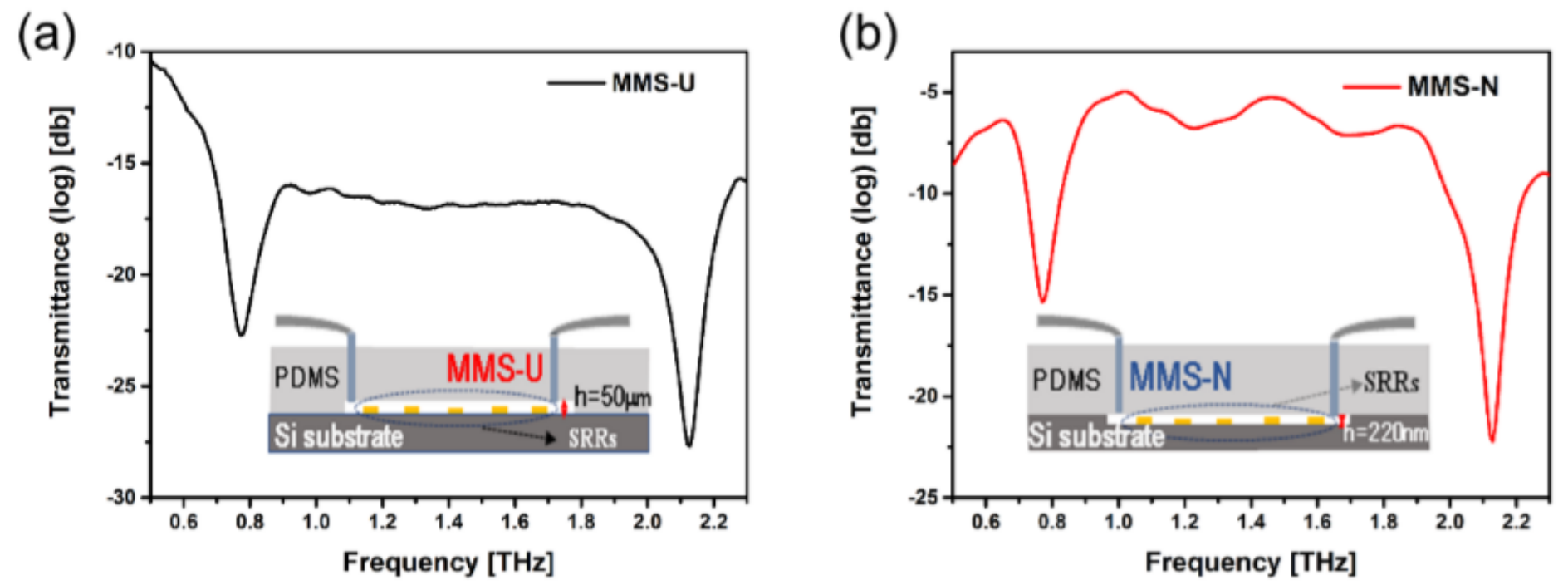
Figure 2

Measured transmission spectra of MMS. (a)Transmission spectra of MMS-U. Inset graph shows schematics of the MMS-U. (b) Transmission spectra of MMS-N. Inset graph shows schematics of the MMS-N.
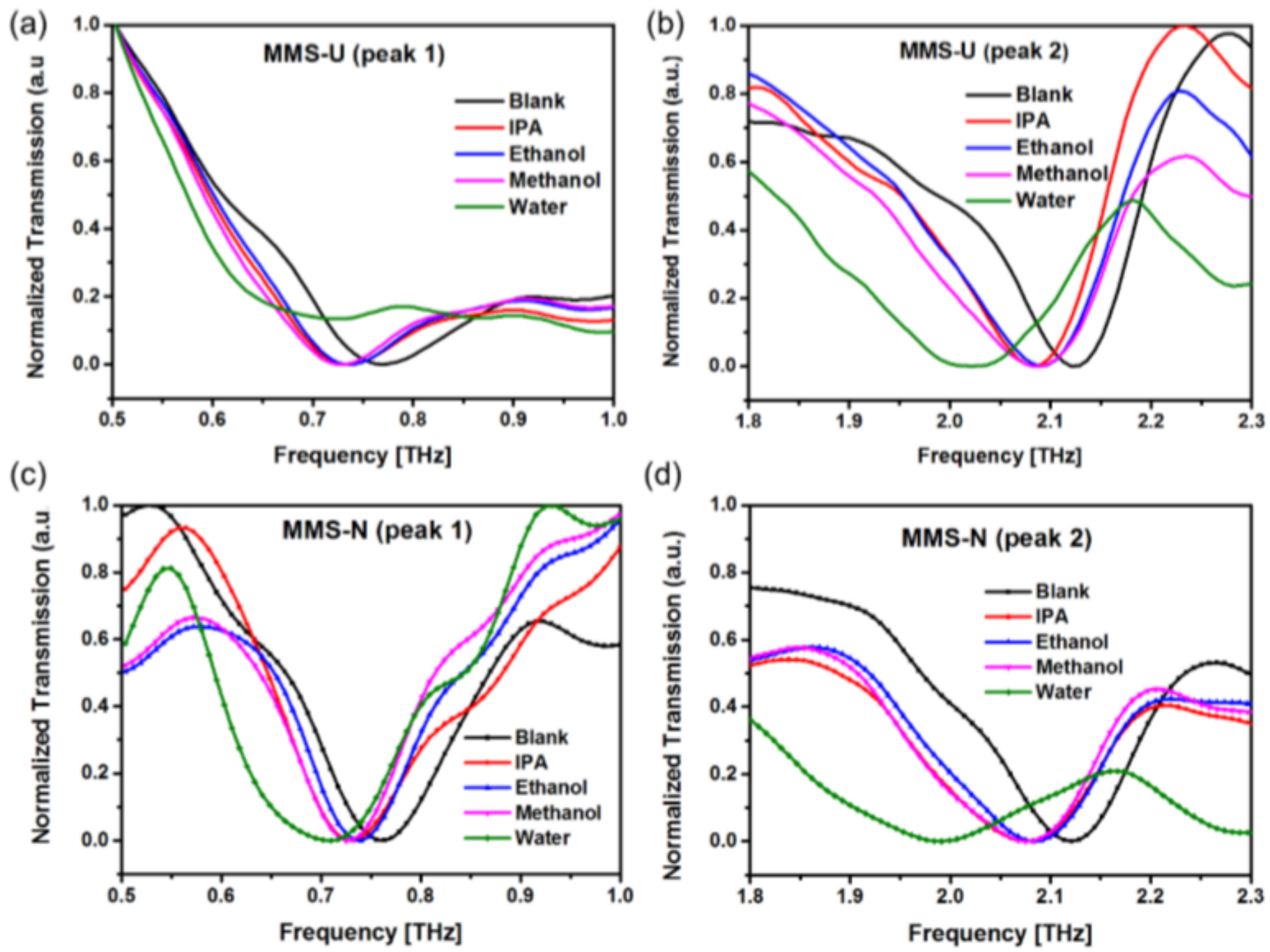

Figure 3

Measured transmission spectra of MMS under different liquid sample environments. Normalized Transmission spectra of peak 1 (a) and peak 2 (b) used MMS-U. Normalized Transmission spectra of peak 1 (c) and peak 2 (d) used MMS-N. 

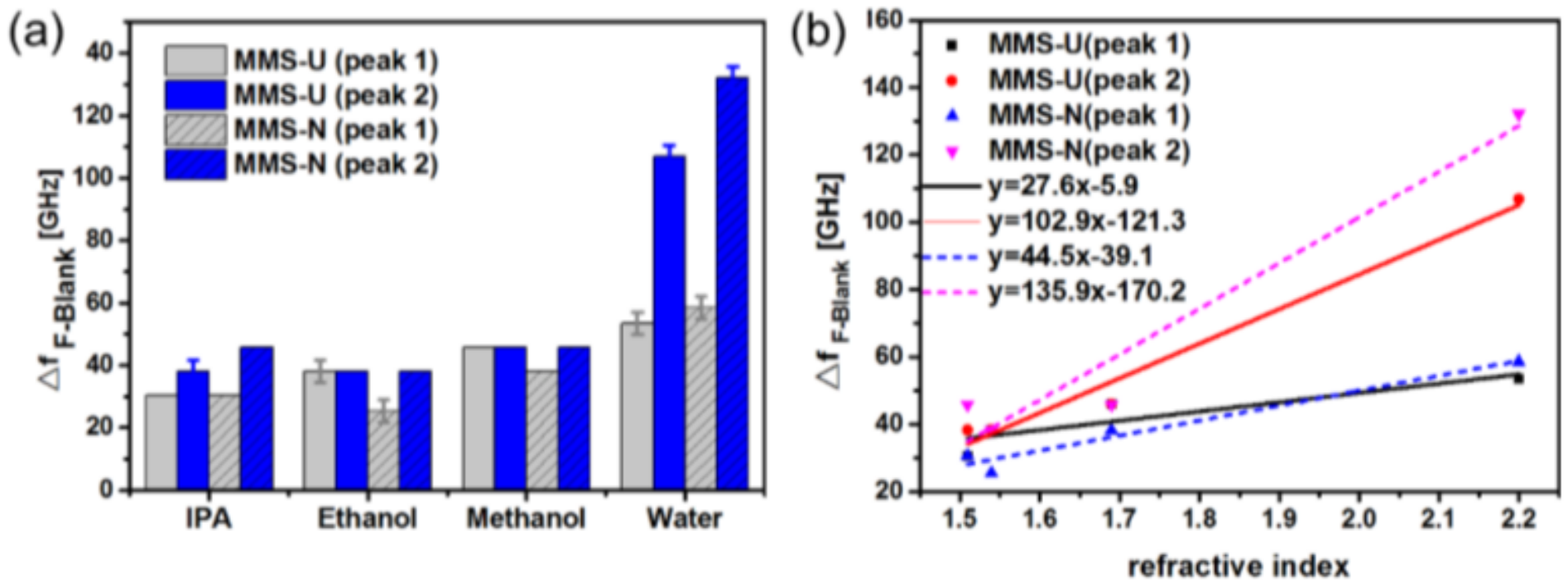

Figure 4

The fixed quantity between the resonant frequency of MMS and different liquid sample. (a) The measured resonance frequency shift with different liquid sample. (b) Fitting of resonance frequency shift with respect to air plotted against the refractive index of each liquid sample.

(a)

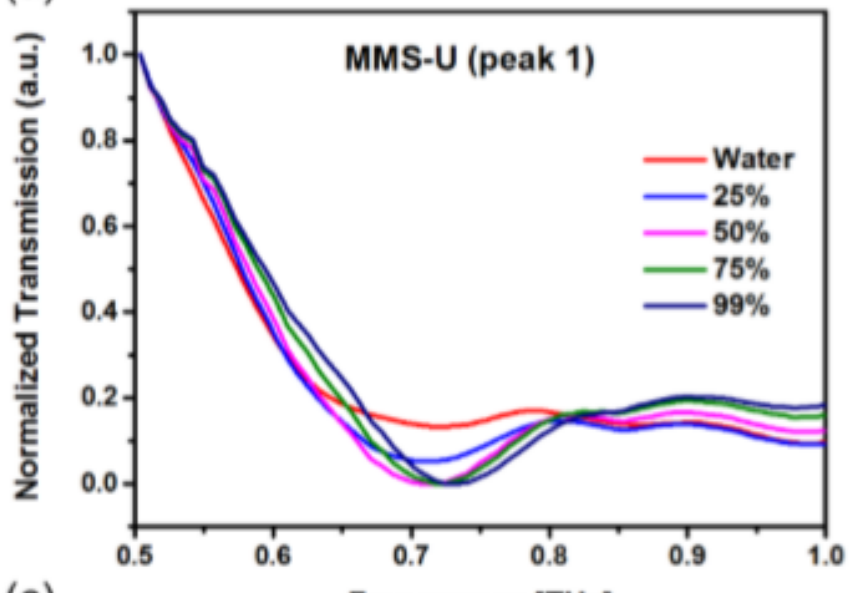

(c)

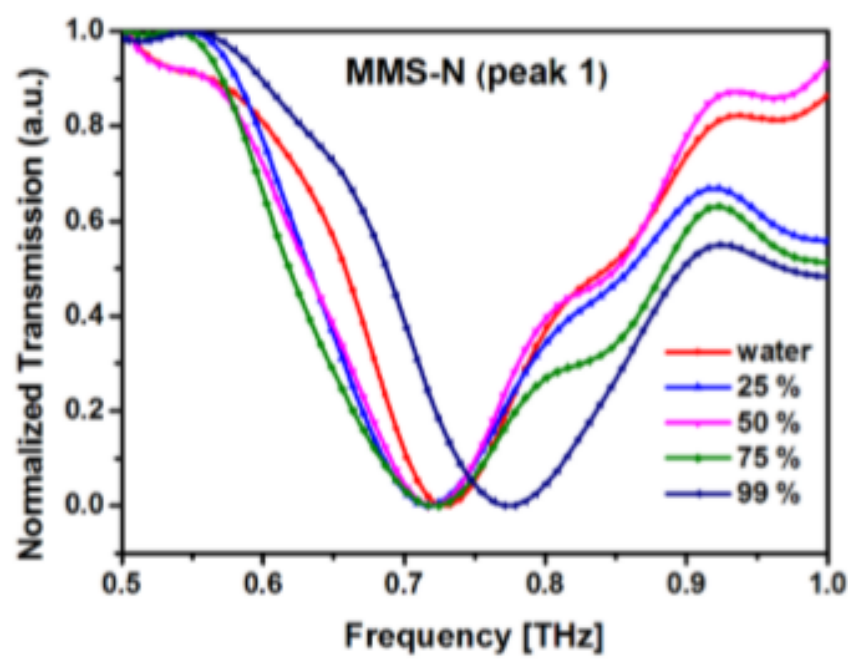

(b)

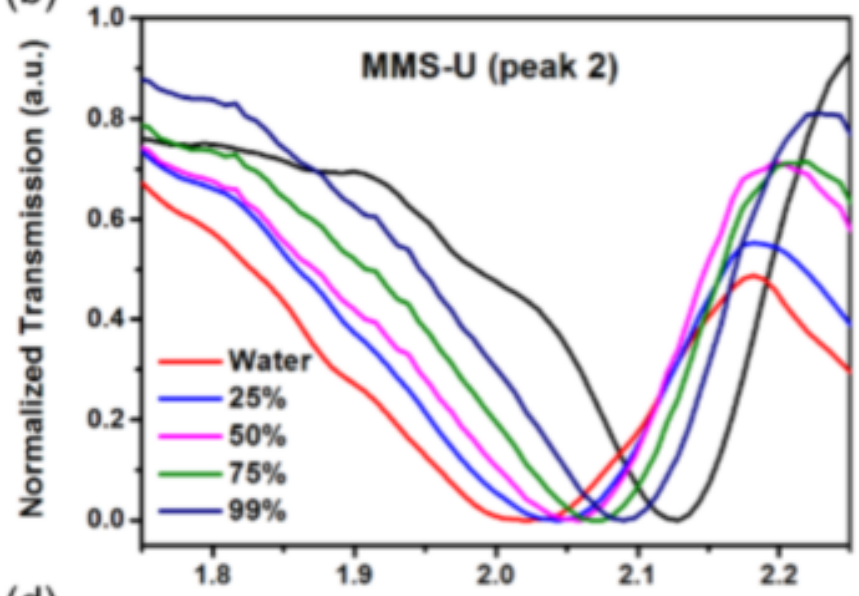

(d)

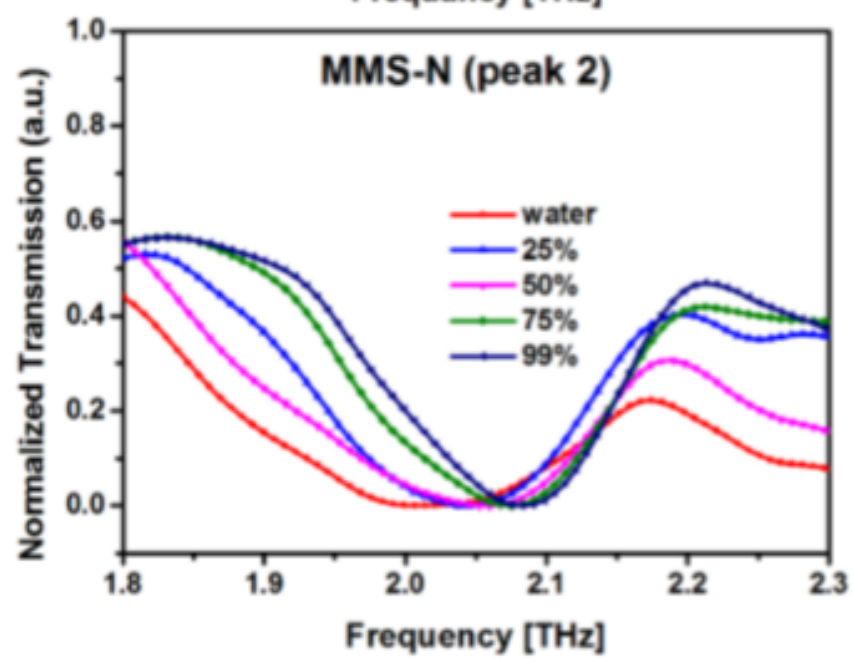




\section{Figure 5}

Measured transmission spectra of MMS with ethanol/water solution of different ethanol concentrations. Normalized transmission spectra of peak 1 (a) and peak 2 (b) using MMS-U with different ethanol concentrations; Measured transmission spectra of peak 1 (c) and peak 2 (d) using MMS-N with of different ethanol concentrations.
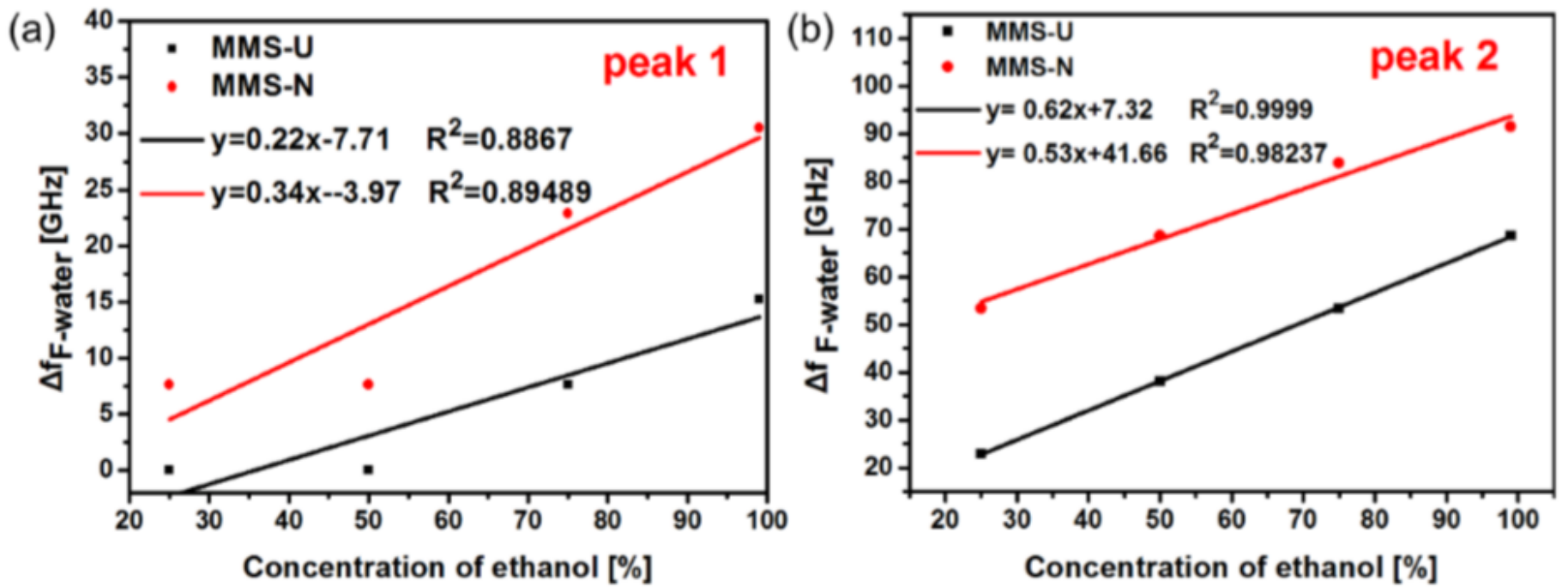

Figure 6

Sensing different concentrations of ethanol. (a) The $\Delta f$ (vs. water) of peak 1 is plotted against different ethanol concentrations. (b) The $\Delta f$ (vs. water) of peak 2 is plotted against different ethanol concentrations.

(a)

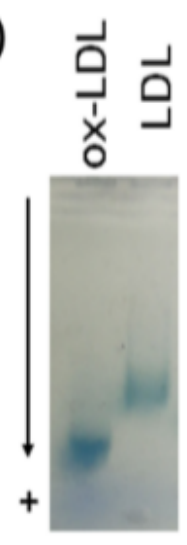

(b)

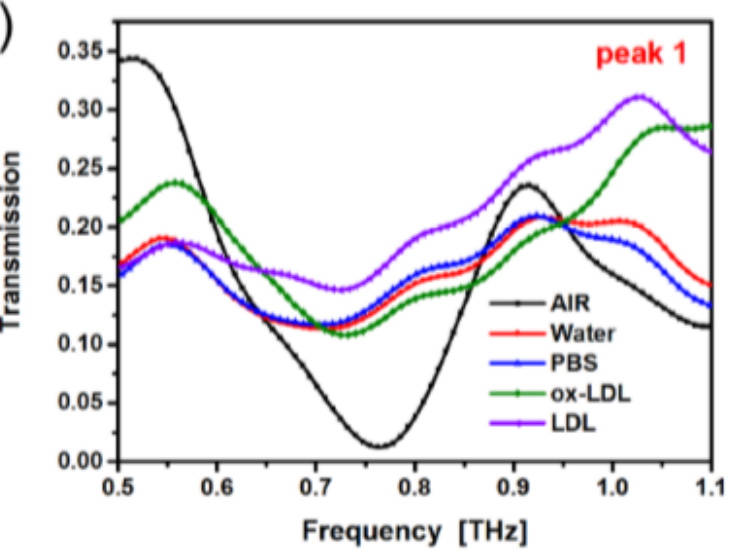

(c)

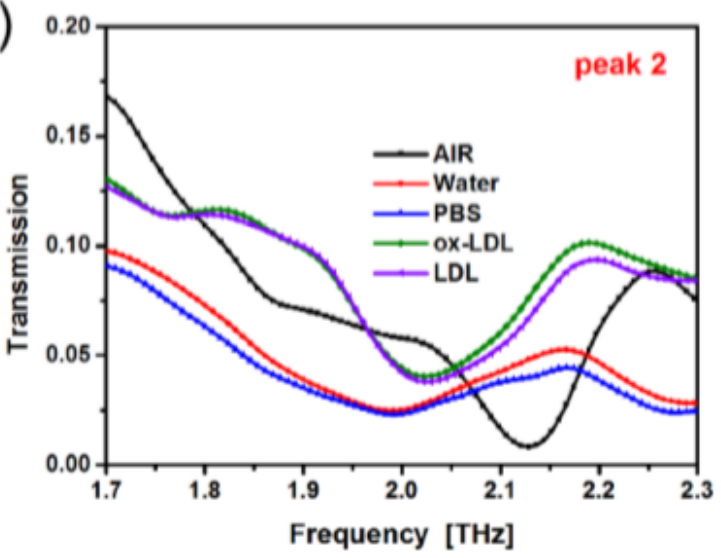

Figure 7

Identification of LDL and ox-LDL. (a)Agarose gel electrophoresis of LDL and ox-LDL; Measured transmission spectra of peak 1 (b) and peak 2 (c) with same concentration of LDL and ox-LDL. 
(a)

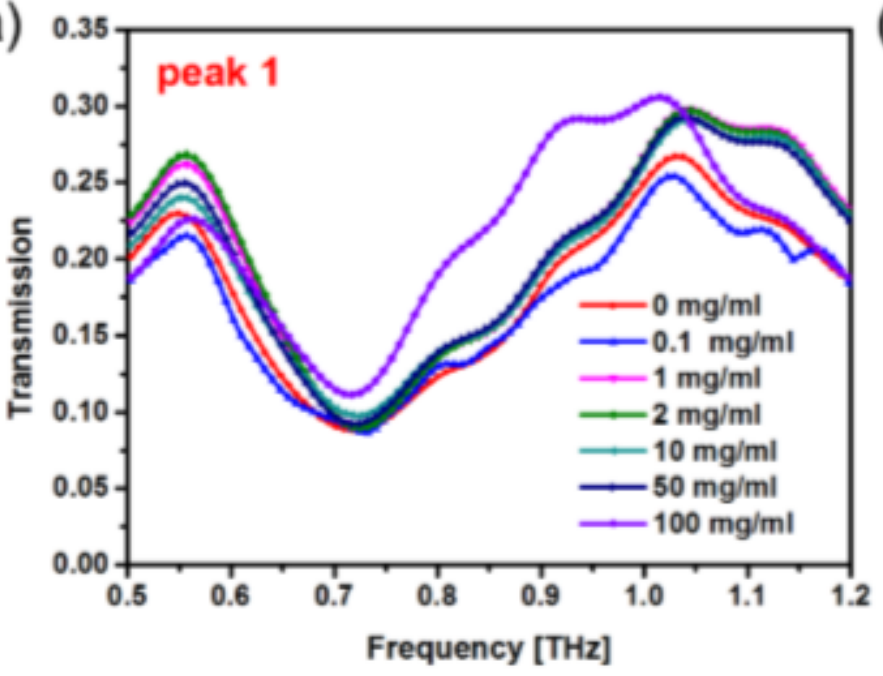

(b)

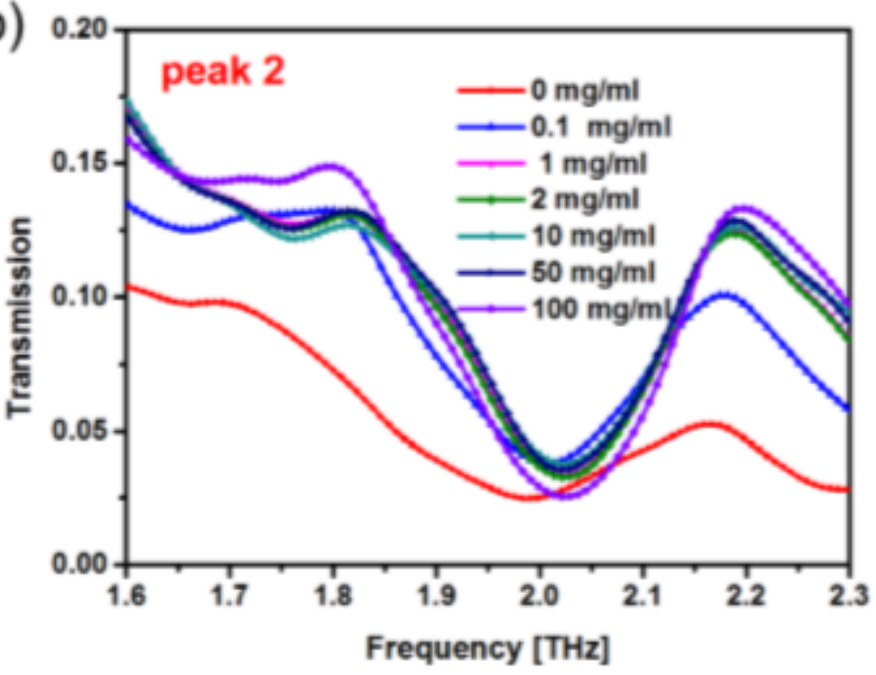

(c)

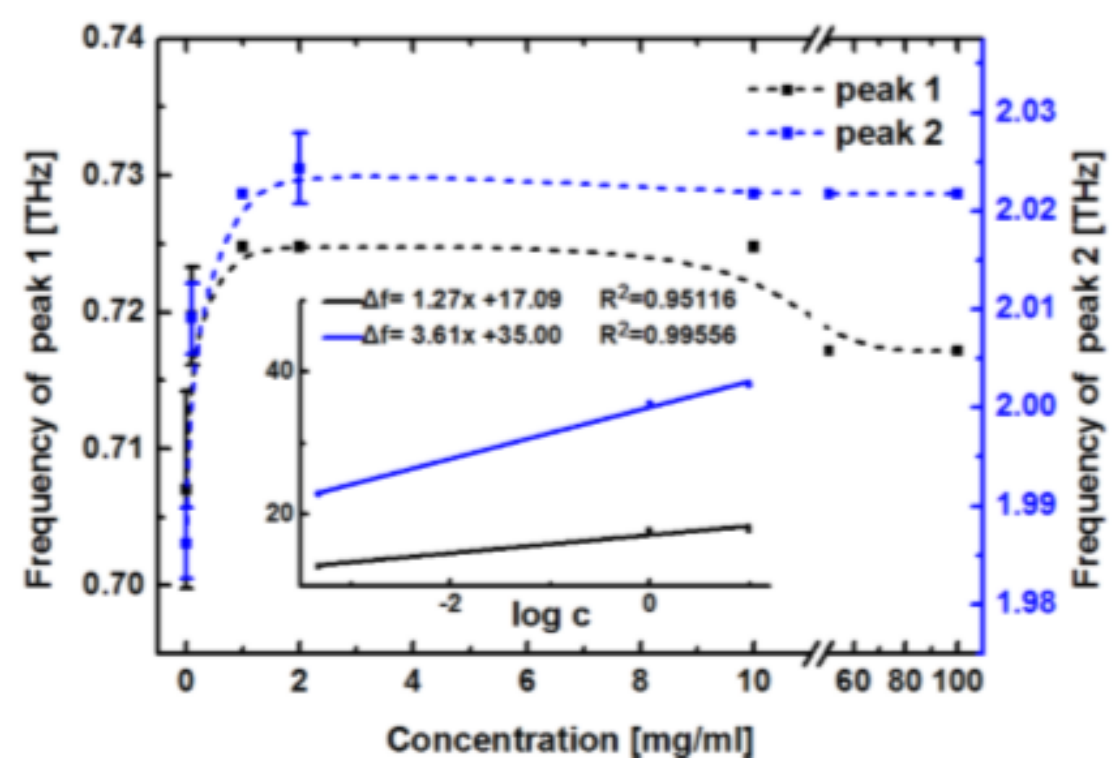

Figure 8

Sensing different concentrations of LDL. Measured transmission spectra of peak 1 (a) and peak 2 (b) with different LDL concentrations. (c) The relation between resonant frequency and solution of concentration for $L D L$. Inset graph shows the relation between $\Delta f$ and solution logarithm of LDL with concentration from 0.1 to $2 \mathrm{mg} / \mathrm{ml}$. 
(a)

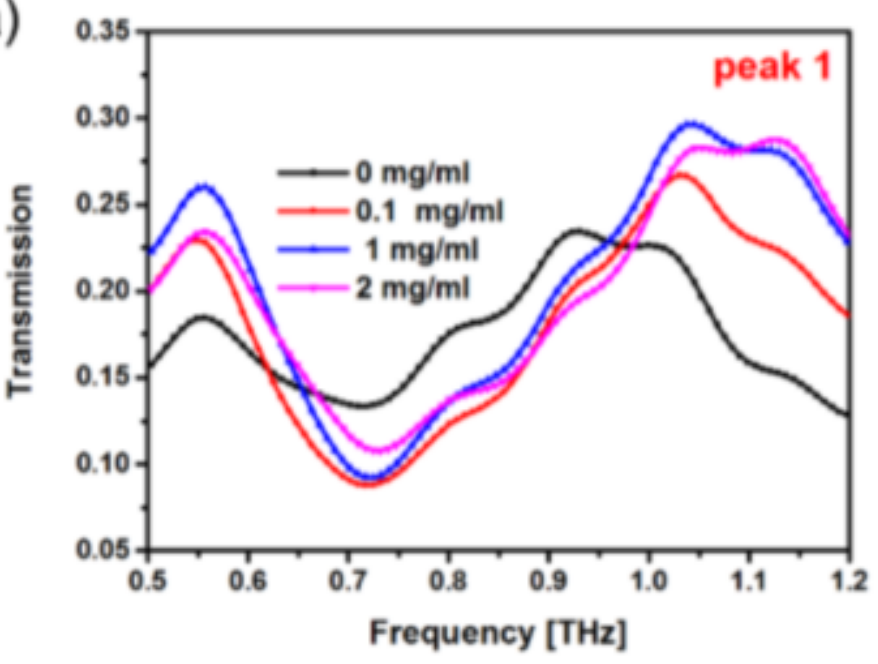

(b)

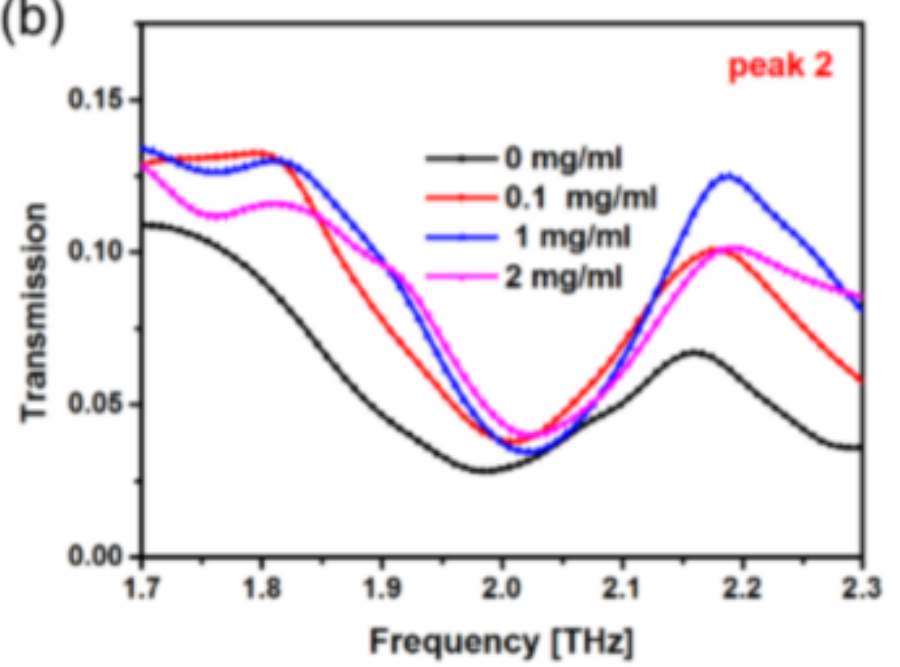

(c)

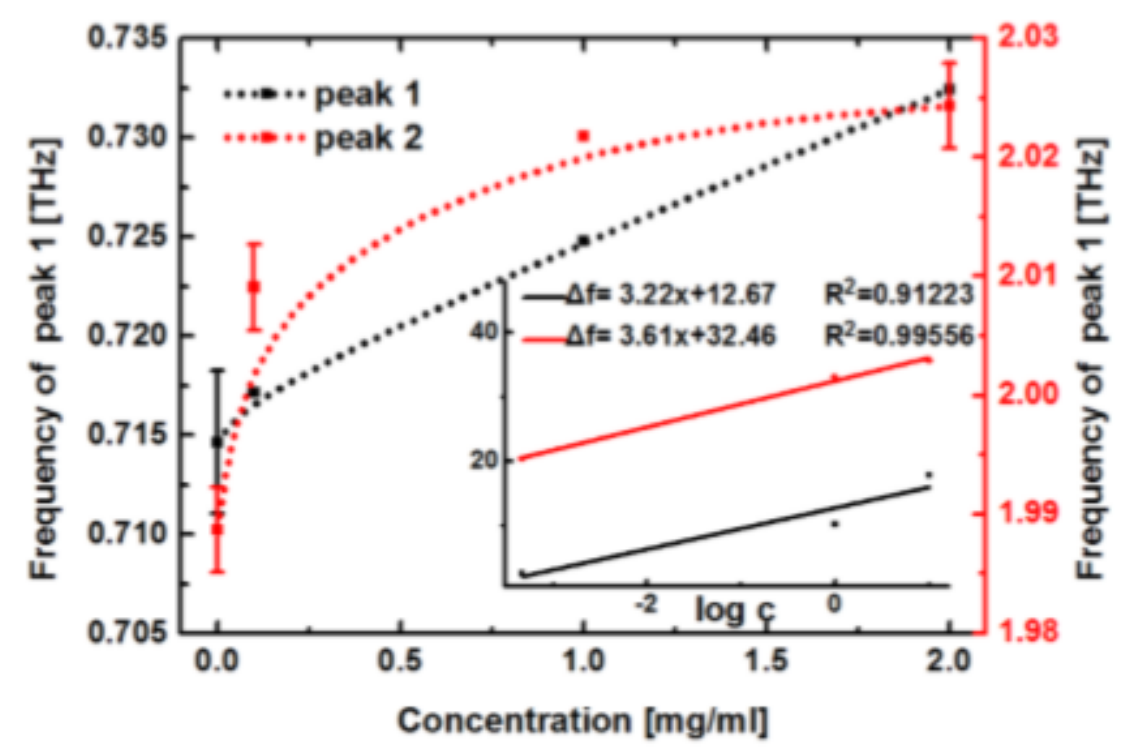

Figure 9

Sensing different concentrations of ox-LDL. Measured transmission spectra of peak 1 (a) and peak 2 (b) with different ox-LDL concentrations. (c) The relation between resonant frequency and solution of concentration for ox-LDL. Inset graph shows the fit of $\Delta f$ to log2C.

\section{Supplementary Files}

This is a list of supplementary files associated with this preprint. Click to download.

- GraphicalAbstract.pdf 